\title{
Mental Health, Smartphone Use Type, and Screen Time Among Adolescents in South Korea
}

\author{
Kyung Soo Woo (iD \\ Su Hyun Bong (D) \\ Tae Young Choi (iD \\ Jun Won Kim
}

Department of Psychiatry, Daegu Catholic University School of Medicine,

Daegu, Republic of Korea
Correspondence: Jun Won Kim Department of Psychiatry, Daegu Catholic University School of Medicine,

33 Duryugongwon-ro 17-gil, Nam-gu,

Daegu, 42472, Republic of Korea

Tel +82-53-650-4332

Fax+82-53-623-1694

Email f_affection@naver.com
Purpose: The excessive use of smartphones for social interaction is associated with mental health. However, few studies have considered the purpose of smartphone usage and screen time together. Therefore, we investigated the impact of smartphone purpose and screen time on mental health using data from the 2017 Korea Youth Risk Behavior Web-based Survey (KYRBS).

Participants and Methods: A total of 54,243 adolescents answered items on purpose and screen time of smartphone use, stress perception, sleep dissatisfaction, depressive symptoms, and suicide-related indicators. The purpose of smartphone usage was categorized as social interaction and non-social interaction, and the daily smartphone screen time was classified into three categories: $<2$ hours, $\geq 2$ hours but $<4$ hours, $\geq 4$ hours per day.

Results: We determined that, on weekdays, stress perception, sleep satisfaction, depressive symptoms, and suicide-related indicators worsened in both groups that used smartphones for $\geq 4$ hours per day. When using smartphone for $\geq 2$ hours but $<4$ hours per day on weekdays, depending on the smartphone use purpose, mental health outcomes differed between the two groups. On weekends, using smartphones for $\geq 2$ hours but $<4$ hours per day worsened sleep satisfaction but decreased suicide-related indicators in both groups. Mental health outcomes improved when participants used smartphones for $\geq 2$ hours but $<4$ hours per day but worsened when smartphones were used for $\geq 4$ hours per day on weekends.

Conclusion: Recommended screen time of smartphone is different depending on the purpose of smartphone use, and the risk of uncontrolled usage is emphasized, rather than the absolute screen time.

Keywords: mental health, smartphone addiction, social interaction, social media

\section{Introduction}

Since the beginning of smartphone popularization in 2007, smartphone ownership has steadily increased globally. As of 2020, more than five billion people possess mobile devices worldwide. In 2018, the ownership rate among South Koreans reached $95 \%$, the highest in the world. ${ }^{1}$ As smartphones become more popular and accessible, interest in the effects of their usage on adolescents is increasing, especially concerning mental health. Thus, the notion of "smartphone addiction" and "problematic smartphone usage" ensued. It refers to prolonged smartphone use, frustration when unable to use a smartphone, and disinterest in non-smartphonerelated activities. These factors indicate addictive behavior and result in impairment of social or other important areas of functioning.

This problematic smartphone usage is associated with the duration of its utilization. $^{2}$ According to a study of 4434 adolescents in $2019,{ }^{3}$ the failure to 
control usage time accounted for the highest percentage of the causes of problematic smartphone use. Rozgonjuk ${ }^{4}$ reports that smartphone usage duration is closely related to problematic smartphone use. In addition, increased duration of smartphone usage in adolescents is related to mental health problems, such as the development of depressive symptoms, ${ }^{5,6}$ decreased general happiness, ${ }^{7}$ poor sleep quality, ${ }^{8}$ suicidal ideation, ${ }^{9}$ and increased risk of obesity and cardiovascular disease. ${ }^{10}$ Considering the risk of excessive smartphone use, the Canadian 24-Hour Movement Guidelines recommends $\leq 2$ hours of screen time for children aged 5-17 years, ${ }^{11}$ and the American Academy of Pediatrics also recommends $<1$ to 2 hours of screen time for children aged 2-18 years. ${ }^{12,13}$

However, certain studies have shown that an excess of the recommended screen time has no negative consequences ${ }^{14}$ or is inconsequential an effect to warrant policy change ${ }^{15}$ on adolescent mental health. This suggests that screen time and mental health may have a complex association and depends on the purpose of usage - for example, reading, playing games, and communicating, and the diversity of media used - rather than a linear relationship. Prior studies found a U-shaped curve between screen time and mental health measures, ${ }^{14,16}$ which increased the risk of depressive symptoms in groups with high screen time but conversely had a positive effect in groups with low or moderate screen time levels. A study of Internet usage duration also confirmed the U-shaped connection to mental health. ${ }^{17,18}$ These results suggest that excessive and uncontrolled use of digital media, rather than absolute screen time, could more negatively affect the mental health of adolescents.

Meanwhile, past studies have primarily focused on the purpose of problematic smartphone usage with regard to the function of social media on smartphones. Smartphone usage for social networking was associated with problematic smartphone use, ${ }^{19}$ depression, low self-esteem, Internet addiction, ${ }^{20}$ substance abuse, ${ }^{21}$ self-harming behavior, ${ }^{22}$ and suicidal ideation, ${ }^{23}$ the most frequent purpose of problematic usage was associated with social interaction ${ }^{24}$ such as social networking service(SNS), messenger, e-mail. In particular, studies have also shown that the use of SNS, such as Facebook and Instagram, is related to depression, anxiety, negative physicality, and alcohol abuse. ${ }^{25}$ However, other studies have reported that using SNS and messenger is not related to problematic smartphone use. ${ }^{26}$ Past studies have begun to categorize smartphone use as social (for social purposes such as SNS or text messaging) and non-social (for non-social purposes such as Internet news, entertainment, and relaxation). ${ }^{27}$ The studies conducted in two categories suggest that the non-social purpose causes more stress, ${ }^{28}$ depression, and anxiety ${ }^{29}$ than social purpose. Therefore, further research on the relevance of the purpose of smartphone usage on mental health outcomes is needed.

Past studies have shown that the screen time and purpose of using smartphones are related to problematic smartphone use, affecting various psychopathology. However, the inconsistent conclusions of studies have rendered them controversial. To the best of our knowledge, no study investigated the association with psychopathology by reflecting both the time and purpose of smartphone use. Therefore, we aimed to verify the difference in the duration spent on smartphones that affects mental health between the two groups when classifying adolescents according to the purpose of smartphone usage. This study categorized the group that uses smartphones for primarily social interaction purposes as the "Social Interaction group" (SI group) and the group that uses them for non-social purposes, such as music and leisure, as the "Non-Social Interaction group" (NSI group). Currently, the Korea Disease Control and Prevention Agency (KDCA) conducts an online survey of 60,000 teenagers annually and uses the data to plan and evaluate adolescent health promotion projects and disclose raw data for research.

\section{Participants and Methods Participants}

The Korea Youth Risk Behavior Web-based Survey (KYRBS) has been conducted annually since 2005 to assess the nationwide prevalence of adolescent healthrisk behaviors by the KDCA. The question consists of a total of about 120 items, including alcohol use, smoking, obesity, and weight control effort, physical activity, dietary behaviors, sexual behaviors, mental health, oral health, personal hygiene, socioeconomic status, etc. The target population for this survey is comprised of nationally representative middle- and high-school students aged 12-18 years in Korea. Approximately 75,000 students from 400 sampled middle and 400 high schools have participated in the survey conducted each year. Students anonymously completed the self-reported questionnaire in a computer laboratory belonging to each sampled school. The current study analyzed data from the 13th survey of KYRBS 
conducted in 2017 since it was the only year that investigated the purpose and screen time of smartphone usage. Ethical approval for analysis of publicly available data used in this study was reviewed by the Institutional Review Board of Daegu Catholic University Hospital (DCUMC IRB approval No. CR-20-228), in accordance with the Declaration of Helsinki. The Institutional Review Board of Daegu Catholic University Hospital exempted this study from requiring ethical approval because all data used and analyzed were anonymous.

Of the 64,991 potential respondents from 800 schools, 62,276 students from 799 schools participated in the survey; the participation rate was $95.8 \%$. When asked, "Have you used your own or someone else's smartphone in the last 30 days?" 54,603 teenagers answered, "Yes." The purpose of smartphone usage is identified by "Choose only one purpose for using the smartphone in the last 30 days." The response items were: (1) Study (online lecture, etc.); (2) Search for information; (3) Use Messenger/Chat (Line, My People, KakaoTalk, etc.); (4) Games; (6) Read/ download cartoon (Webtoon) or novel (Web Novel); (7) Listen to/download music; (8) Using UCC/Video (Africa TV, YouTube, etc.); (9) Using cafes/communities; (10) Using e-mail; (11) Shopping online; (12) SNS (blogs, Instagram, Twitter, Facebook, etc.); and (13) Other. () Regarding the question about the purpose of using smartphones, the respondents who answered using options (3), (9), (10), and (12) were categorized as belonging to the SI group, and the remaining respondents were categorized as belonging to the NSI group. Item (13) was excluded from the analysis because no details were specified. Finally, 54,243 respondents were included in the analysis of this study.

\section{Measurements}

Sociodemographic variables extracted from the raw data included age, sex, area of residence, socio-economic status (SES), and academic achievement. The area of residence was divided into large cities, small cities, and rural areas. SES and academic achievement were categorized as "high," "medium-high," "medium," "medium-low," and "low." In the present study, "high" and "medium-high" were combined to denote high SES and academic achievements, and "medium-low" and "low" were combined to denote low SES and academic achievement.

The smartphone screen time for weekdays (Monday to Friday) and weekends (Saturday to Sunday) were measured separately with the question "For how many hours have you used your smartphone on average in the last 30 days?" Participants' responses were in 30-minute increments (ie, 30 minutes, 1 hour, 1 hour 30 minutes, etc.). The average daily screen time of smartphones was classified into three categories: " $<2$ hours," " $\geq 2$ hours but $<4$ hours," and " $\geq 4$ hours," considering prior studies. ${ }^{30,31}$

Mental health outcomes included stress perception, sleep dissatisfaction, depression, suicidal ideation, a suicide plan, and suicidal attempt history. Stress perception was evaluated using the question "How much stress do you usually experience?" The responses were recorded on a five-point Likert-type scale: (1) very much; (2) a lot; (3) a little; (4) very little; (5) none at all. Choosing options (1) or (2) indicated that participants were stressed and choosing any of the other three options did not. Sleep dissatisfaction was evaluated using the question "Do you think the time spent sleeping in the last 7 days was sufficient for fatigue recovery?" and assessed with the options: "(1) very sufficient; (2) sufficient; (3) just adequate; (4) not enough; and (5) not enough at all." Options (1) and (2) indicated satisfaction with the amount of sleep, and the other three options did not. Depressive symptoms, suicidal ideation, a suicide plan, and suicide attempt history were evaluated by: "Have you felt so sad or hopeless in the last 12 months that you have stopped your daily life for two weeks?"; "In the last 12 months, have you seriously considered committing suicide?"; "In the last 12 months, have you made any specific plans to commit suicide?"; and, "Have you attempted suicide in the last 12 months?" respectively. The questions were evaluated by the responses "yes" and "no."

\section{Statistical Analysis}

This study aimed to classify the purpose of using smartphones through the 13th online survey of the KYRBS conducted in 2017. Moreover, it sought to identify the association between the two groups' weekday and weekend smartphone screen time and purpose to evaluate the mental health of adolescents.

Based on the analytical guidelines of the KDCA, complex sample weights were applied to reflect nationally representative samples. Descriptive statistics were used to identify sociodemographic and mental health characteristics according to the purpose of smartphone usage of the participants. Independent $t$-tests and Rao-Scott chi-square tests were used to compare sociodemographic variables, smartphone screen time, and mental health outcomes between the two groups. A complex sample logistic regression was 
performed to calculate the odds ratios (OR) ${ }^{32}$ and the $95 \%$ confidence interval (CI) of mental health by smartphone screen time and purpose of the participants after adjusting for sex, age, SES, and academic achievement. All statistical analyses were performed using the SPSS 25.0 software (IBM, Armonk, NY, 2017). The significance level was set at a p-value $<0.05$.

\section{Results}

\section{Comparison of Sociodemographic} Characteristics and Mental Health Scale

Of the total participants, the SI group comprised 25,740 (47.5\%) participants, and the NSI group comprised 26,582 (52.5\%) participants. The mean ages of the SI and NSI groups were $15.20 \pm 0.03$ years and $15.08 \pm 0.03$ years, respectively. There were significant differences in age, sex, academic achievement, and socioeconomic status. The ratio of female adolescents and was higher in the SI group. On weekdays, the distribution according to daily smartphone screen time of the SI group was categorized as follows: $21.1 \%$ used smartphones for $<2$ hours; $41.5 \%$ used smartphones for $\geq 2$ hours but $<4$ hours; and 37.4\% used smartphones for $\geq 4$ hours. The distribution according to daily smartphone screen time of the NSI group was categorized as follows: $31.6 \%$ used smartphones for $<2$ hours; $42.7 \%$ used smartphones for $\geq 2$ hours but $<4$ hours; and $25.7 \%$ used smartphones for $\geq 4$ hours. On weekends, distribution according to smartphone screen time of the SI group was categorized as follows: $7.5 \%$ used smartphones for $<2$ hours; $24.4 \%$ used smartphones for $\geq 2$ hours but $<4$ hours; and $68.1 \%$ used smartphones for $\geq 4$ hours. The distribution according to daily smartphone screen time of the NSI group was categorized as follows: $14.2 \%$ used smartphones for $<2$ hours; $30.4 \%$ used smartphones for $\geq 2$ hours but $<4$ hours; and $55.3 \%$ used smartphones for $\geq 4$ hours (Table 1). There were statistically significant differences in the SI group apropos of stress perception, sleep dissatisfaction, depression, suicidal thoughts, and suicidal history $(\mathrm{p}<0.001)$. There was no significant difference between the two groups apropos of a suicide plan (Table 2).

\section{Relationship Between Smartphone Screen Time and Mental Health Outcomes, According to the Purpose of Smartphone Use on Weekdays}

When using a smartphone for $\geq 4$ hours during the week, in the SI group, risk of stress perception (OR, 1.216; 95\%
CI, 1.135-1.302), sleep dissatisfaction (OR, 1.103; 95\% CI, 1.008-1.208), depression (OR, 1.385; 95\% CI, 1.2751.504), suicidal ideation (OR, 1.248; 95\% CI, 1.1341.374), suicide plan (OR, 1.231; 95\% CI, 1.027-1.476), past history of suicide (OR, 1.437; 95\% CI, 1.146-1.801) increased. When using a smartphone for $\geq 4$ hours during the week, in the NSI group, the risk of stress perception (OR, 1.156; 95\% CI, 1.079-1.239), sleep dissatisfaction (OR, 1.141; 95\% CI, 1.050-1.240), depression (OR, 1.289; 95\% CI, 1.192-1.393), suicidal ideation (OR, 1.315; 95\% CI, 1.181-1.465), suicide plan (OR, 1.344; 95\% CI, 1.136-1.592), and past history of suicide (OR, 1.547 ; 95\% CI, 1.236-1.937) increased. When smartphones were used for $\geq 2$ hours but $<4$ hours per day, sleep dissatisfaction (OR, 1.074; 95\% CI, 1.005-1.146), depression (OR, 1.113; 95\% CI, 1.039-1.194) significantly increased in the NSI group, but no mental health outcomes increased in the SI group (Table 3).

\section{Relationship Between Smartphone Screen Time and Mental Health Outcomes, According to the Purpose of Smartphone Use on Weekends}

When the screen time of smartphone was $\geq 2$ hours but $<4$ hours, the risk of sleep dissatisfaction (OR, 1.154; 95\% CI, 1.018-1.309) in the SI group increased, but it decreased the risk of past history of suicide (OR, 0.620; 95\% CI, 0.439-0.874). Contrarily, in the NSI group, the risk of sleep dissatisfaction (OR, 1.134; 95\% CI, 1.044-1.232) increased, but reduced stress perception (OR, 0.910; 95\% CI, 0.838-0.988), suicidal ideation (OR, 0.877; 95\% CI, 0.773-0.995), suicide plan (OR, 0.665; 95\% CI, 0.5470.808 ), and past history of suicide (OR, $0.738 ; 95 \% \mathrm{CI}$, $0.558-0.975)$. If the screen time of smartphone use was $\geq 4$ hours, the risk of sleep dissatisfaction (OR, 1.331; 95\% CI, 1.185-1.494) and depressive symptoms (OR, 1.288 ; $95 \%$ CI, 1.149-1.444) increased in the SI group. In the NSI group, stress perception (OR, 1.111; 95\% CI, 1.027-1.202), sleep dissatisfaction (OR, 1.300; 95\% CI, 1.199-1.409), and depression (OR, 1.115; 95\% CI, 1.0191.219) increased and the risk of suicide plan (OR, 0.803; 95\% CI, 0.677-0.953) decreased (Table 4).

\section{Discussion}

The purpose of this study was to identify the connection between smartphone screen time and mental health based on the purpose of smartphone usage. The study was 
Table I General Characteristics and Smartphone Usage Duration in Each Group Classified According to Purpose

\begin{tabular}{|c|c|c|c|c|}
\hline \multirow[t]{2}{*}{ Variables } & Total $(n=54,243)$ & SI Group $(n=25,740)$ & NSI Group $(n=28,503)$ & \multirow[t]{2}{*}{ p-value } \\
\hline & Mean \pm SD or N(\%) & Mean \pm SD or N(\%) & Mean \pm SD or N(\%) & \\
\hline Age (years) & $15.14 \pm 0.02$ & $15.20 \pm 0.03$ & $15.08 \pm 0.03$ & $<0.00 I^{*}$ \\
\hline Sex & & & & $<0.00 I^{*}$ \\
\hline Male & $26,725(50.5)$ & $893 I(36.2)$ & $17,794(63.3)$ & \\
\hline Female & $27,5 \mid 8(49.5)$ & $16,809(63.8)$ & $10,709(36.7)$ & \\
\hline Area of residence & & & & 0.632 \\
\hline Rural & $3028(4.6)$ & |428(4.4) & $1707(4.7)$ & \\
\hline Small city & $22,242(43.8)$ & $10,867(44.2)$ & $12,159(43.5)$ & \\
\hline Large city & $27,052(51.6)$ & $\mid 3,445(5 \mid .4)$ & $14,637(5 \mid .8)$ & \\
\hline Socio-economic status & & & & $<0.00 I^{*}$ \\
\hline Low & $1302(2.4)$ & $595(2.4)$ & $707(2.4)$ & \\
\hline Low-middle & $642 I(11.6)$ & $2944(\mid 1.2)$ & $3477(12.1)$ & \\
\hline Middle & $25,155(46.0)$ & $12,069(46.6)$ & $13,086(45.5)$ & \\
\hline High-middle & $15,9 \mid 8(29.7)$ & 7634(29.8) & $8284(29.5)$ & \\
\hline High & $5447(10.2)$ & 2498(9.9) & $2949(10.5)$ & \\
\hline Academic achievement & & & & $<0.00 I^{*}$ \\
\hline Low & $5042(9.4)$ & $2514(10.0)$ & $2528(8.8)$ & \\
\hline Low-middle & $\mathrm{II}, 825(2 \mathrm{I} .8)$ & $5854(22.9)$ & $597 \mid(20.9)$ & \\
\hline Middle & $|5,62|(29.0)$ & $7466(29.1)$ & $8155(28.8)$ & \\
\hline High-middle & $14,354(26.3)$ & $6756(25.9)$ & $7598(26.7)$ & \\
\hline High & $740 I(I 3.5)$ & $3150(12.1)$ & $425 I(I 4.8)$ & \\
\hline Smartphone screen time on weekdays & & & & $<0.00 I^{*}$ \\
\hline$<2$ hours & $14,428(26.7)$ & $54 \mid 2(21.1)$ & $9016(31.6)$ & \\
\hline$\geq 2$ hours but $<4$ hours & $22,612(42.1)$ & $10,58 \mid(4 \mid .5)$ & $|2,03|(42.7)$ & \\
\hline$\geq 4$ hours & $17,203(31.2)$ & $9747(37.4)$ & $7456(25.7)$ & \\
\hline Smartphone screen time on weekends & & & & $<0.00 I^{*}$ \\
\hline$<2$ hours & $5829(\mid 1.0)$ & $1863(7.5)$ & $3966(14.2)$ & \\
\hline$\geq 2$ hours but $<4$ hours & $14,589(27.6)$ & $6073(24.4)$ & $8516(30.4)$ & \\
\hline$\geq 4$ hours & $33,825(61.4)$ & $17,804(68.1)$ & $|6,02|(55.3)$ & \\
\hline
\end{tabular}

Notes: Weighted percentages following complex sample analysis. ${ }^{p}<0.001$.

conducted using a large sample of Korean adolescents. Concerning the weekday smartphone screen time, both SI and NSI groups had experienced increased risk in their stress perception, sleep dissatisfaction, depression, suicidal ideation, a suicide plan, and history of suicide when smartphone screen time was $\geq 4$ hours. Using a smartphone for $\geq 2$ hours but $<4$ hours per day during the weekday, there was no mental health variable that experienced an increased risk in the SI group, but the risk of sleep dissatisfaction and depression in the NSI group increased. This means that using a smartphone for social purposes during the week for less than 4 hours did not increase the risk of mental health problems, even if it exceeded the conventional screen time guideline of 2 hours a day. However, in the case of using smartphones for non-social purposes during the week, when the average screen time was 2 hours or more, mental health variables deteriorated. Thus, using smartphones for social purposes acts as a protective factor against mental health problems since there are Internet functions that can strengthen social support, connection, sense of belonging, and self-expression. This is a different result from the existing guidelines, which present digital media such as TVs as a risk associated with obesity and cardiovascular diseases, considering the sedentary lifestyle habits that go along with it. ${ }^{13}$ Thus, it suggests that smartphones need new cutting points considering usage purposes and habits. 
Table 2 Self-Reported Mental Health of Participants According to the Purpose of Smartphone Use

\begin{tabular}{|c|c|c|c|c|}
\hline \multirow[t]{2}{*}{ Variables } & Total $(n=54,243)$ & SI Group $(n=25,740)$ & NSI Group $(n=28,503)$ & \multirow[t]{2}{*}{ p-value } \\
\hline & $\mathbf{N}(\%)$ & $\mathbf{N}(\%)$ & $\mathbf{N}(\%)$ & \\
\hline Stress perception & & & & $<0.00 I^{*}$ \\
\hline High & $20,427(37.5)$ & $10,244(39.5)$ & $10,183(35.7)$ & \\
\hline Low & $33,816(62.5)$ & $15,496(60.5)$ & $18,320(64.3)$ & \\
\hline Sleep dissatisfaction & & & & $<0.00 I^{*}$ \\
\hline Yes & $40,7 \mid 7(75.9)$ & $20,470(80.2)$ & $20,247(72.0)$ & \\
\hline No & $13,526(24.1)$ & $5270(19.8)$ & $8256(28.0)$ & \\
\hline Depressive symptoms & & & & $<0.00 I^{*}$ \\
\hline Yes & $13,730(25.4)$ & $7418(28.8)$ & $6312(22.2)$ & \\
\hline No & $40,513(74.6)$ & $\mid 8,322(7 \mid .2)$ & $22,191(77.8)$ & \\
\hline Suicidal ideation & & & & $<0.00 I^{*}$ \\
\hline Yes & $6647(12.2)$ & $3458(13.3)$ & $3189(11.1)$ & \\
\hline No & $47,596(87.8)$ & $22,282(86.7)$ & $25,3 \mid 4(88.9)$ & \\
\hline Suicide plan & & & & 0.054 \\
\hline Yes & $2072(3.8)$ & $1026(3.9)$ & $1046(3.6)$ & \\
\hline No & $52,17 \mid(96.2)$ & $24,7 \mid 4(96.1)$ & $27,457(96.4)$ & \\
\hline Suicide attempt & & & & $<0.00 I^{*}$ \\
\hline Yes & $|36|(2.5)$ & $719(2.7)$ & $642(2.2)$ & \\
\hline No & $52,882(97.5)$ & $25,02 I(97.3)$ & $27,86 \mid(97.8)$ & \\
\hline
\end{tabular}

Notes: Weighted percentages following complex sample analysis. ${ }^{*} \mathrm{p}<0.00$ I.

Weekend smartphone screen time for $\geq 4$ hours increased the risks of stress perception, sleep dissatisfaction, and depression for both groups, while the same reduced the risk of suicide plan in the NSI group. Regarding suicide planning, it is not only the opposite of other mental health variables but it is also inconsistent with the results of a previous study. ${ }^{9}$ However, further interpretation of the results of the suicide plan question, along with further research, is needed. In the case of smartphone screen time for $\geq 2$ hours but $<4$ hours on weekends, the risk of sleep dissatisfaction increased in both groups. The association between insufficient sleep and screen time of smartphones in bed has been reported in previous studies, ${ }^{33,34}$ which revealed that the use of electronic devices for more than 2 hours had a direct relationship with sleep. ${ }^{35}$

It is noteworthy that the smartphone screen time for $\geq 2$ hours but $<4$ hours on weekends reduced the risk of past history of suicide in the SI group and reduced the risk of stress perception, suicidal ideation, a suicide plan, and past history of suicide in the NSI group. The risk of mental health variables that reduced when smartphones were used for $\geq 2$ hours but $<4$ hours on weekends were found to increase in both groups when smartphone screen time exceeded 4 hours on weekdays. This is similar to the results of previous studies that reported the existing screen time or Internet usage, and the mental health variables do not have a simple linear relationship. According to these results, the use of smartphones for an appropriate time can have a positive effect on mental health. It suggests that uncontrolled use rather than absolute screen time is related more to mental health. In the case of adolescents, education on smartphone use and supervision to ensure appropriate screen time will be necessary; this will help them develop their own schedule of using it, considering that their ability of self-control is still in the developmental stage. In addition, considering the social situation of teenagers whose daily lives change depending on whether they go to school or not, ${ }^{36}$ appropriate smartphone screen time may vary between weekdays and weekends.

The current study has certain strengths. First, prior studies have been conducted on local or smaller samples, whereas this study has selected participants through composite sampling for a large-scale of adolescent samples, and the weights are taken into account in the analysis. Second, in this study, the purpose of using the smartphone and screen time were considered together and analyzed to further investigate the relationship between mental health 
Table 3 Associations of Self-Reported Mental Health with the Smartphone Purpose and Screen Time on Weekdays

\begin{tabular}{|c|c|c|}
\hline \multirow[t]{2}{*}{ Variables } & SI Group & NSI Group \\
\hline & OR (95\% Cl) & OR (95\% Cl) \\
\hline \multicolumn{3}{|l|}{ Stress perception } \\
\hline$<2$ hours & Reference & Reference \\
\hline$\geq 2$ hours but $<4$ hours & $1.029(0.963-1.099)$ & $0.985(0.929-1.044)$ \\
\hline$\geq 4$ hours & $1.216(1.135-1.302)$ & $1.156(1.079-1.239)$ \\
\hline \multicolumn{3}{|l|}{ Sleep dissatisfaction } \\
\hline$<2$ hours & Reference & Reference \\
\hline$\geq 2$ hours but $<4$ hours & $0.986(0.904-1.075)$ & $1.074(1.005-1.146)$ \\
\hline$\geq 4$ hours & $1.103(1.008-1.208)$ & $1.141(1.050-1.240)$ \\
\hline \multicolumn{3}{|l|}{ Depressive symptoms } \\
\hline$<2$ hours & Reference & Reference \\
\hline$\geq 2$ hours but $<4$ hours & $1.014(0.937-1.098)$ & $1.113(1.039-1.194)$ \\
\hline$\geq 4$ hours & $1.385(1.275-1.504)$ & $1.289(1.192-1.393)$ \\
\hline \multicolumn{3}{|l|}{ Suicidal ideation } \\
\hline$<2$ hours & Reference & Reference \\
\hline$\geq 2$ hours but $<4$ hours & $0.942(0.854-1.039)$ & $1.066(0.973-1.168)$ \\
\hline$\geq 4$ hours & $1.248(1.134-1.374)$ & $1.315(1.181-1.465)$ \\
\hline \multicolumn{3}{|l|}{ Suicide plan } \\
\hline$<2$ hours & Reference & Reference \\
\hline$\geq 2$ hours but $<4$ hours & $0.924(0.774-1.102)$ & $0.968(0.83 I-1.128)$ \\
\hline$\geq 4$ hours & 1.231 (1.027-1.476) & $1.344(1.136-1.592)$ \\
\hline \multicolumn{3}{|l|}{ Suicide attempt } \\
\hline$<2$ hours & Reference & Reference \\
\hline$\geq 2$ hours but $<4$ hours & $0.902(0.720-1.130)$ & $1.003(0.817-1.233)$ \\
\hline$\geq 4$ hours & $1.437(1.146-1.801)$ & $1.547(1.236-1.937)$ \\
\hline
\end{tabular}

Notes: Odds ratio $(\mathrm{OR})$ and $95 \%$ confidence interval $(\mathrm{Cl})$ was estimated with multivariate logistic regression analysis adjusting for sex, age, socio-economic status, and academic achievement. All the variables presented $p$-value $<0.00$ I. "Reference" indicates the reference time for comparing the OR. Significant ORs are highlighted in bold.

outcomes and smartphone usage. Third, considering the lifestyle related to school attendance in adolescents, smartphone usage time was classified into weekdays and weekends and surveyed, suggesting a difference in the degree of smartphone screen time on mental health according to lifestyle.

Nonetheless, this study also has a few limitations. First, the online survey on youth health behavior is conducted every year since 2005 in Korea, but only the 2017 KYRBS survey was related to smartphone use. Therefore, since a cross-sectional study design was used, the temporal and causal relationship between the actual purpose of smartphone use, screen time, and mental health outcomes could not be confirmed. Second, the questions related to the mental health outcomes are insufficient to authenticate a clinical diagnosis, and the questions used a relatively simple two-point Likert scale. When a past study evaluated the reliability of KRYBS' mental health items, a suicidal attempt item had substantial reliability $($ kappa $=0.70)$, and other mental health items had at least moderate reliability (kappas ranged 0.50 to 0.58 ). ${ }^{37,38}$ However, future work is required in assessing the validity of self-report measures of mental health items. Third, as a self-reported questionnaire, it is necessary to consider that a recall bias may occur in questions about mental health in the recent year, and the objective standards for academic performance and SES are insufficient. Since the questionnaire adheres to only one main service purpose, asking adolescents the purpose of using smartphones and the criticism of using a smartphone may be partially mixed. Finally, since no previous study has proved the absolute recommendation criteria for adolescent smartphone screen time, the basis for the unit time used in this study has not been clearly established. On this basis, 
Table 4 Associations of Self-Reported Mental Health with the Smartphone Purpose and Screen Time on Weekends

\begin{tabular}{|c|c|c|}
\hline \multirow[t]{2}{*}{ Variables } & SI Group & NSI Group \\
\hline & OR $(95 \% \mathrm{CI})$ & OR $(95 \% \mathrm{CI})$ \\
\hline \multicolumn{3}{|l|}{ Stress perception } \\
\hline$<2$ hours & Reference & Reference \\
\hline$\geq 2$ hours but $<4$ hours & $1.058(0.948-1.180)$ & $0.910(0.838-0.988)$ \\
\hline$\geq 4$ hours & $1.186(1.069-1.315)$ & $1.111(1.027-1.202)$ \\
\hline \multicolumn{3}{|l|}{ Sleep dissatisfaction } \\
\hline$<2$ hours & Reference & Reference \\
\hline$\geq 2$ hours but $<4$ hours & $1.154(1.018-1.309)$ & $1.134(1.044-1.232)$ \\
\hline$\geq 4$ hours & 1.331 (1.185-I.494) & $1.300(1.199-1.409)$ \\
\hline \multicolumn{3}{|l|}{ Depressive symptoms } \\
\hline$<2$ hours & Reference & Reference \\
\hline$\geq 2$ hours but $<4$ hours & $1.087(0.963-1.227)$ & $0.942(0.854-1.038)$ \\
\hline$\geq 4$ hours & $1.288(1.149-1.444)$ & 1.115 (1.019-1.219) \\
\hline \multicolumn{3}{|l|}{ Suicidal ideation } \\
\hline$<2$ hours & Reference & Reference \\
\hline$\geq 2$ hours but $<4$ hours & 0.861 (0.743-I.009) & $0.877(0.773-0.995)$ \\
\hline$\geq 4$ hours & I.083 (0.94I-I.246) & $1.122(0.999-1.260)$ \\
\hline \multicolumn{3}{|l|}{ Suicide plan } \\
\hline$<2$ hours & Reference & Reference \\
\hline$\geq 2$ hours but $<4$ hours & $0.802(0.615-1.046)^{*}$ & $0.665(0.547-0.808)$ \\
\hline$\geq 4$ hours & $0.934(0.732-1.192)^{*}$ & $0.803(0.677-0.953)$ \\
\hline \multicolumn{3}{|l|}{ Suicide attempt } \\
\hline$<2$ hours & Reference & Reference \\
\hline$\geq 2$ hours but $<4$ hours & $0.620(0.439-0.874)$ & $0.738(0.558-0.975)$ \\
\hline$\geq 4$ hours & $0.87 \mid(0.647-I .17 \mid)$ & $1.03 \mid(0.8 \mid 2-1.308)$ \\
\hline
\end{tabular}

Notes: Odds ratio $(\mathrm{OR})$ and $95 \%$ confidence interval $(\mathrm{Cl})$ was estimated with multivariate logistic regression analysis adjusting for sex, age, socio-economic status, and academic achievement. All the variables presented p-value $<0.00$ I, except *p-value $>0.05$. "Reference" indicates the reference time for comparing the OR. Significant ORs are highlighted in bold.

the authors set the criteria in consideration of the guidelines and the previous studies relating to existing screen time recommendations.

\section{Conclusion}

Among adolescents using smartphones for $\geq 2$ hours but $<$ 4 hours per day on weekdays, the change in the mental health outcomes was different depending on the purpose they were used for. When using smartphones for $\geq 4$ hours per day on weekdays, all mental health measures worsened in both groups. As for weekends, when using the smartphone for $\geq 2$ hours but $<4$ hours per day, the risk of sleep dissatisfaction increased, but the risk of suiciderelated indicators decreased in both groups. However, the risk of mental health outcomes increased again when screen time was $\geq 4$ hours. This suggests that recommended screen time of smartphones is different depending on the purpose they are used for, and the risk associated with uncontrolled usage is higher than that of absolute screen time. Since the mental health status of adolescents is at a critical stage for the development of psychopathology, specific smartphone guidelines and preventive measures should be prepared that fully consider the lifestyle of adolescents and the purpose and screen time of smartphone usage. In the future, additional longitudinal studies should be conducted to confirm the causality between smartphones usage and mental health outcomes.

\section{Abbreviations}

KYRBS, Korea Youth Risk Behavior Web-based Survey; SNS, Social Networking Service; SI group, Social Interaction group; NSI group, Non-Social Interaction group; OR, Odds ratio. 


\section{Acknowledgments}

We would like to thank Editage (www.editage.co.kr) for

English language editing.

\section{Disclosure}

The authors report no conflicts of interest in this work.

\section{References}

1. Silver L. Smartphone ownership is growing rapidly around the world, but not always equally. Washington, D.C.: Pew Research Center; 2019. Available from: https://www.pewresearch.org/global/2019/02/ 05/smartphone-ownership-is-growing-rapidly-around-the-world-butnot-always-equally/. Accessed June 1, 2021.

2. Lee H, Kim JW, Choi TY. Risk factors for smartphone addiction in Korean adolescents: smartphone use patterns. J Korean Med Sci. 2017;32(10):1674-1679. doi:10.3346/jkms.2017.32.10.1674

3. National Information Society Agency. 2019 The survey on Smartphone overdependence (NIA VIII-RSE-C-19067). Daegu; 2019. Available from: https://www.nia.or.kr/site/nia_kor/ex/bbs/ View.do?cbIdx $=65914 \& b c I d x=21939 \&$ parentSeq $=21939$. Accessed June 1, 2021.

4. Rozgonjuk D, Levine JC, Hall BJ, Elhai JD. The association between problematic smartphone use, depression and anxiety symptom severity, and objectively measured smartphone use over one week. Comput Human Behav. 2018;87:10-17. doi:10.1016/j.chb.2018.05.019

5. Riehm KE, Feder KA, Tormohlen KN, et al. Associations between time spent using social media and internalizing and externalizing problems among US youth. JAMA Psychiatr. 2019;76 (12):1266-1273. doi:10.1001/jamapsychiatry.2019.2325

6. Lemola S, Perkinson-Gloor N, Brand S, Dewald-Kaufmann JF, Grob A. Adolescents' electronic media use at night, sleep disturbance, and depressive symptoms in the smartphone age. $J$ Youth Adolesc. 2015;44(2):405-418. doi:10.1007/s10964-014-0176-x

7. Helliwell J, Layard R, Sachs J. World happiness report 2019. New York: Sustainable Development Solutions Network; 2019. Available from: https://worldhappiness.report/ed/2019/. Accessed June 1, 2021.

8. Buxton OM, Chang A-M, Spilsbury JC, Bos T, Emsellem H, Knutson KL. Sleep in the modern family: protective family routines for child and adolescent sleep. Sleep Health. 2015;1(1):15-27. doi:10.1016/j.sleh.2014.12.002

9. Luxton DD, June JD, Fairall JM. Social media and suicide: a public health perspective. Am J Public Health. 2012;102(S2):S195-S200.

10. Robinson TN, Banda JA, Hale L, et al. Screen media exposure and obesity in children and adolescents. Pediatrics. 2017;140(Suppl 2): S97-S101. doi:10.1542/peds.2016-1758K

11. Tremblay MS, Carson V, Chaput J-P, et al. Canadian 24-hour movement guidelines for children and youth: an integration of physical activity, sedentary behaviour, and sleep. Appl Physiol Nutr Metabol. 2016;41(6):S311-S327. doi:10.1139/apnm-2016-0151

12. Communications Co, Media. Children, adolescents, and the media. Pediatrics. 2013;132(5):958-961. doi:10.1542/peds.2013-2656

13. Communications Co, Media, MBE. Media use in school-aged children and adolescents. Pediatrics. 2016;138(5):e20162592. doi:10. 1542/peds.2016-2592

14. Przybylski AK, Weinstein N. A large-scale test of the goldilocks hypothesis: quantifying the relations between digital-screen use and the mental well-being of adolescents. Psychol Sci. 2017;28 (2):204-215. doi:10.1177/0956797616678438

15. Orben A, Przybylski AK. The association between adolescent well-being and digital technology use. Nat Human Behav. 2019;3 (2):173-182. doi:10.1038/s41562-018-0506-1
16. Liu M, Wu L, Yao S. Dose-response association of screen time-based sedentary behaviour in children and adolescents and depression: a meta-analysis of observational studies. Br J Sports Med. 2016;50 (20):1252-1258. doi:10.1136/bjsports-2015-095084

17. Bélanger RE, Akre C, Berchtold A, Michaud P-A. A U-shaped association between intensity of Internet use and adolescent health. Pediatrics. 2011;127(2):e330-e335. doi:10.1542/peds.2010-1235

18. Moreno MA, Jelenchick L, Koff R, Eickhoff J. Depression and Internet use among older adolescents: an experience sampling approach. Psychology. 2012;3(09):743. doi:10.4236/psych.2012.32 9112

19. Lopez-Fernandez O, Kuss DJ, Romo L, et al. Self-reported dependence on mobile phones in young adults: a European cross-cultural empirical survey. J Behav Addict. 2017;6(2):168-177. doi:10.1556/ 2006.6.2017.020

20. Pantic I. Online social networking and mental health. Cyberpsychol Behav Soc Netw. 2014;17(10):652-657. doi:10.10 89/cyber.2014.0070

21. Gallimberti L, Buja A, Chindamo S, et al. Problematic cell phone use for text messaging and substance abuse in early adolescence (11-to 13-year-olds). Eur J Pediatr. 2016;175(3):355-364. doi:10.1007/ s00431-015-2645-y

22. Dyson MP, Hartling L, Shulhan J, et al. A systematic review of social media use to discuss and view deliberate self-harm acts. PLoS One. 2016;11(5):e0155813. doi:10.1371/journal.pone.0155813

23. Frost RL, Rickwood DJ. A systematic review of the mental health outcomes associated with Facebook use. Comput Human Behav. 2017;76:576-600. doi:10.1016/j.chb.2017.08.001

24. Tossell C, Kortum P, Shepard C, Rahmati A, Zhong L. Exploring smartphone addiction: insights from long-term telemetric behavioral measures. Int J Interact Mobile Technol. 2015;9(2):37-43. doi:10. 3991/ijim.v9i2.4300

25. Jeong S-H, Kim H, Yum J-Y, Hwang Y. What type of content are smartphone users addicted to?: SNS vs. games. Comput Human Behav. 2016;54:10-17. doi:10.1016/j.chb.2015.07.035

26. Bae S-M. The relationship between the type of smartphone use and smartphone dependence of Korean adolescents: national survey study. Child Youth Serv Rev. 2017;81:207-211. doi:10.1016/j. childyouth.2017.08.012

27. Song I, Larose R, Eastin MS, Lin CA. Internet gratifications and Internet addiction: on the uses and abuses of new media. Cyberpsychol Behav. 2004;7(4):384-394. doi:10.1089/cpb.2004.7. 384

28. Van Deursen AJ, Bolle CL, Hegner SM, Kommers PA. Modeling habitual and addictive smartphone behavior: the role of smartphone usage types, emotional intelligence, social stress, self-regulation, age, and gender. Comput Human Behav. 2015;45:411-420. doi:10.1016/j. chb.2014.12.039

29. Rozgonjuk D, Elhai JD, Täht K, Vassil K, Levine JC, Asmundson GJ. Non-social smartphone use mediates the relationship between intolerance of uncertainty and problematic smartphone use: evidence from a repeated-measures study. Comput Human Behav. 2019;96:56-62. doi:10.1016/j.chb.2019.02.013

30. Long J, Liu T-Q, Liao Y-H, et al. Prevalence and correlates of problematic smartphone use in a large random sample of Chinese undergraduates. BMC Psychiatr. 2016;16(1):408. doi:10.1186/ s12888-016-1083-3

31. Robinson M, Kendall GE, Jacoby P, et al. Lifestyle and demographic correlates of poor mental health in early adolescence. J Paediatr Child Health. 2011;47(1-2):54-61. doi:10.1111/j.1440-1754.2010.01 891.x

32. Currie C, Molcho M, Boyce W, Holstein B, Torsheim T, Richter M. Researching health inequalities in adolescents: the development of the Health Behaviour in School-Aged Children (HBSC) family affluence scale. Soc Sci Med. 2008;66(6):1429-1436. doi:10.1016/j. socscimed.2007.11.024 
33. Sampasa-Kanyinga H, Hamilton HA, Chaput JP. Use of social media is associated with short sleep duration in a dose-response manner in students aged 11 to 20 years. Acta Paediatrica. 2018;107 (4):694-700. doi:10.1111/apa.14210

34. Carter B, Rees P, Hale L, Bhattacharjee D, Paradkar MS. Association between portable screen-based media device access or use and sleep outcomes: a systematic review and meta-analysis. JAMA Pediatr. 2016;170(12):1202-1208. doi:10.1001/jamapediatrics.2016.2341

35. Twenge JM, Krizan Z, Hisler G. Decreases in self-reported sleep duration among US adolescents 2009-2015 and association with new media screen time. Sleep Med. 2017;39:47-53. doi:10.1016/j. sleep.2017.08.013
36. Wittmann M, Dinich J, Merrow M, Roenneberg T. Social jetlag: misalignment of biological and social time. Chronobiol Int. 2006;23 (1-2):497-509. doi:10.1080/07420520500545979

37. Bae J, Joung H, Kim JY, et al. Test-retest reliability of a questionnaire for the Korea Youth Risk Behavior Web-based Survey. J Prev Med Public Health. 2010;43:403-410. doi:10.3961/jpmph.2010.43.5.403

38. Park S-W, Joung H, Kim J-Y, Bae J, Kwon KN. Reliability and validity of the Korea youth risk behavior web-based survey questionnaire. Osong City: Korea Centers for Disease Control and Prevention; 2009. Available from: https://scienceon.kisti.re.kr/srch/ selectPORSrchReport.do?cn=TRKO201300000170. Accessed June $1,2021$.
Psychology Research and Behavior Management

\section{Publish your work in this journal}

Psychology Research and Behavior Management is an international, peer-reviewed, open access journal focusing on the science of psychology and its application in behavior management to develop improved outcomes in the clinical, educational, sports and business arenas. Specific topics covered in the journal include: Neuroscience, memory and decision making; Behavior modification and management; Clinical

\section{Dovepress}

applications; Business and sports performance management; Social and developmental studies; Animal studies. The manuscript management system is completely online and includes a very quick and fair peer-review system, which is all easy to use. Visit http://www. dovepress.com/testimonials.php to read real quotes from published authors. 